\title{
Plant Health 2020 Abstracts of Special Session Presentations
}

Special Session Abstracts submitted for presentation at the APS Plant Health 2020 Annual Meeting held virtually, August 10-14, 2020. The recommended format for citing annual meeting abstracts, using the first abstract below as an example, is as follows:

Groover, W., Lawrence, K. S., Held, D., and Carson, K. 2020. Plant growth-promoting rhizobacteria: A novel management strategy for Meloidogyne spp. on turfgrass. (Abstr.) Phytopathology 110:S2.207. https://doi.org/10.1094/PHYTO-110-12-S2.207.

The abstracts are published as a supplement to Phytopathology for citation purposes. They were not reviewed by the Phytopathology Editorial Board and were not edited by the APS editorial staff.

https://doi.org/10.1094/PHYTO-110-12-S2.207

(C) 2020 The American Phytopathological Society

Plant growth-promoting rhizobacteria: A novel management strategy for Meloidogyne spp. on turfgrass W. GROOVER, K. S. Lawrence, D. Held, K. Carson, Entomology and Plant Pathology, Auburn University, Auburn, AL, USA

Meloidogyne spp., root-knot nematodes, are among the most economically important plant-parasitic nematodes in turfgrass in the United States. Only a few nematicides are available or efficacious for plant-parasitic nematodes in turfgrass in the United States, and recent work has demonstrated the potential for microbial control of root-knot nematodes in field crops. The objectives of this study were to evaluate the efficacy of 104 plant growth-promoting rhizobacteria (PGPR) strains isolated from grasses in Alabama against $M$. incognita in vitro, and their ability to manage plant-parasitic nematodes in the greenhouse and microplot settings. In vitro mortality ranged from 0.9 to $98.9 \%$ mortality by all PGPR strains screened. Ten individual PGPR strains and one three-strain blend (13 total PGPR strains) were advanced to greenhouse and microplot screening. In the greenhouse, six of the 11 PGPR treatments significantly reduced $M$. incognita population density, with multiple strains also promoting root growth. In the microplot, five of the 11 PGPR treatments significantly reduced $M$. incognita population density, while again promoting root growth. Of these strains, 11 were identified as Bacillus spp., one as Stenotrophomonas rhizophila, and one as Paenibacillus sonchi. Eight of these strains were also found to have nitrogenase activity, and seven have the ability to produce siderophores, showing a potential mechanism for growth promotion. Overall, results indicate that multiple strains of Bacillus spp. and one strain of S. rhizophila have potential to reduce M. incognita population density and enhance turfgrass root growth.

Genome-based circumscription of cool-virulent Ralstonia solanacearum strains in an attempt to improve their regulation P. SHARMA, Genetics, Bioinformatics, and Computational Biology program, Virginia Tech, Blacksburg, VA, USA

Ralstonia solanacearum $(R s)$ is a plant pathogen that causes disease on hundreds of plant species including staple crops such as potato and banana. A small group of Rs strains, known as Race 3 Biovar 2 (R3B2), cause potato brown rot disease at cooler temperatures and lead to crop losses of up to $100 \%$. R3B2 strains do not occur in the USA and because of the ease of transmission and cool-virulence, they pose a serious threat to the US potato industry. Since it is challenging to distinguish R3B2 strains from other Rs strains and also because R3B2 strains are not a formally described taxon, the entire Rs species, including many Rs strains endemic to the US, is included in the list of highly regulated select agents. To help USDA APHIS improve the regulation of cool-virulent $R s$ stains, the aim of this study is to apply a genome-based classification scheme capable of precisely identifying and distinguishing the cool-virulent R3B2 strains from non-cool virulent $R s$ strains. Towards this goal, a core genome phylogenetic tree of over $100 R s$ strains was constructed, including 18 confirmed and putative cool-virulent strains. This tree clearly separated cool-virulent strains from other $R s$ strains. Next, a genome-based distance tree was constructed and genome similarity-based Life Identification Numbers (LINs) were assigned to each Rs strain using the web server LINbase. Both trees and the assigned LINs were found to group the cool-virulent strains similarly. Genomebased classification and identification of cool-virulent $R s$ strains is thus possible. However, virulence assays of putative cool-virulent strains still need to be performed to precisely define which strains should be included in a revised select agent definition. Once this is done, USDA APHIS will have a new tool in hand to protect US agriculture from cool-virulent $R s$ strains. 
A Global Surveillance System (GSS) for crop diseases: Global preparedness minimizes the risk to food supplies

M. CARVAJAL-YEPES (1), K. F. Cardwell (2), A. Nelson (3), K. A. Garrett (4), B. Giovani (5), D. G. O. Saunders (6), S. Kamoun (7), J. P. Legg (8), V. M. Verdier (9), J. Lessel (10), R. A. Neher (11), R. Day (12), S. Senait (13), P. Pardey (14), M. L. Gullino (15), A. R. Records (16), B. R. Bextine (17), J. E. Leach (18), S. Staiger (1), J. Tohme (1), (1) Alliance of Bioversity and CIAT, Cali, COLOMBIA; (2) Oklahoma State University, Stillwater, OK, USA; (3) University of Twente, Enschede, NETHERLANDS; (4) Plant Pathology Department, University of Florida, Gainesville, FL, USA; (5) European and Mediterranean Plant Protection OrganizationEuphresco, Paris, FRANCE; (6) John Innes Centre, Norwich, UNITED KINGDOM; (7) The Sainsbury Laboratory, Norwich, UNITED KINGDOM; (8) International Institute of Tropical Agriculture, Dar es Salaam, TANZANIA; (9) IRD, Cirad, Univ Montpellier, IPME, Montpellier, FRANCE; (10) Gro-Intelligence, NY, USA; (11) University of Basel, SWITZERLAND; (12) Centre for Agriculture and Biosciences International, Wallingford, UNITED KINGDOM; (13) University of Minnesota, Saint Paul, MN, USA; (14) University of Minnesota, St Paul, MN, USA; (15) University of Torino, Grugliasco Torino, ITALY; (16) USAID, Washington, DC, USA; (17) Univ of Texas At Tyler, Tyler, TX, USA; (18) Colorado State University, Fort Collins, CO, USA

A Global Surveillance System (GSS) is proposed to provide global advance awareness of where pests and diseases are causing problems, inform food security assessments, and enable more coordinated and effective intervention. The GSS would be comprised of existing surveillance systems worldwide, but with increased efficacy achieved through deliberate coordination of people, compilation and analysis of disease diagnostic data patterns, and a forward-looking goal of improved risk management at a global scale. Creating linkages between general and specific surveillance entities across countries, the GSS would increase coordination in high-consequence disease detection, allowing optimization of early response and control. It would function through five interconnected networks that all share a cross-cutting capacity-development component: (i) diagnostic labs and specialists, (ii) risk assessment and decision support modeling teams, (iii) data standardization and management specialists, (iv) regular expert communications, and (v) a distributed operations management system. A pilot phase would focus on high-risk diseases causing high economic impact in some of the world's most important crops (maize, potato, cassava, rice, beans, and wheat) in low-income countries. Successful implementation of the GSS will require collaboration, communication and integration of existing national and international diagnostic networks, including the international CGIAR Germplasm Health Units (GHUs) that support regional pest surveillance and ensure safe germplasm distribution for many crop-breeding programs.

\author{
Modulation of temperatures impact on disease resistance by light quality, ethylene and nuclear pore complex in Arabidopsis \\ thaliana \\ J. HUA, Cornell Univ, Ithaca, NY, USA
}

Temperature has a large impact on plant immune responses. I will present and discuss recent studies from my lab on the modulation of temperature impact on resistance to Pseudomonas syringae in Arabidopsis thaliana by light quality, ethylene, and nuclear pore complex (NPC). 1) We examined the interactive effects of light qualities (white, red, and blue) and growth temperatures $\left(16^{\circ} \mathrm{C}, 22^{\circ} \mathrm{C}\right.$, and $28^{\circ} \mathrm{C}$ ) on disease resistance. We found that the effects of temperature are dependent on light quality and revealed the role of PHYB and CRY under blue light and red light respectively. 2) We analyzed the effects of moderately low temperature $\left(16^{\circ} \mathrm{C}\right)$ on disease resistance. We found that it enhances disease resistance through the SA pathway. Multiple SA biosynthesis and signaling genes are upregulated at lower temperature. Ethylene represses SA signaling via direct bindings of the ethylene transcription factors to the promoters of multiple SA biosynthesis and signaling genes. 3) We analyzed the role nuclear pore complex (NPC) in high temperature $\left(28^{\circ} \mathrm{C}\right)$ inhibition of disease resistance. We found that the outer ring subunits of NPC have a non-redundant temperature-influenced function in NLR mediated disease resistance. Both mRNA export and nuclear retention of transcription factors are preferentially compromised at high temperature with the loss of function of some NPC subunits. This temperature-influenced NPC activities likely contribute to the high temperature inhibition of disease resistance.

\title{
Pathogen inoculum monitoring for real-time disease management decisions
} L. D. THIESSEN, North Carolina State University, Raleigh, NC, USA

Disease management decisions by farmers may be based on first visual detections of plant disease, visual estimations of disease severity, or calendar-based fungicide application schedules, which can limit fungicide efficacy and disease control. Inoculum detection tools are valuable resources for improving these management decisions to reduce economic losses from unnecessary or untimely fungicide applications. For example, fungicide applications for grape powdery mildew control were initiated at first detection of inoculum and subsequent applications were timed on using spore thresholds. Additionally, inoculum detection technologies can be used for monitoring fungicide resistance development to be used for targeted disease management strategies. These tools are being further adapted to other pathosystems that reduce immediate and long-term costs to producers and improve the sustainability of disease management.

\section{Breaking Boundaries: Perspectives on the Future of Plant Virology} E. CIENIEWICZ, Clemson University, Clemson, SC, USA

The majority of new and emerging plant pathogens are viruses. Both old and new viruses continue to threaten crop production worldwide, and effects of climate change on viruses and their vectors are imminent yet largely unknown. In the present and the future, plant virologists need to 1) Break down disciplinary boundaries to approach problems and create solutions, 2) Embrace 'omic' technologies to study virus movement and evolution on a landscape scale-between cultivated crops and wild hosts, as well as in multiple infections, and 3) Shift the paradigm of reactive responses to plant virus emergence to proactive and curative approaches. Here I will discuss examples of virus transmission between wild and cultivated perennial hosts-ilarviruses of Prunus spp. (Prunus necrotic ringspot 
virus [PNRSV] and prune dwarf virus [PDV]) and grapevine red blotch virus (GRBV) of Vitis spp. PNRSV and PDV are transmitted via infected pollen, whereas GRBV is transmitted by a treehopper, Spissistilus festinus. These viruses are found in wild hosts near commercial plantings, but the implications of this and their potential for spread differ depending on their respective dispersal mechanisms, landscape composition, and biotic and abiotic interactions. Research progress to date, future research directions, and implications on the management of the respective virus diseases are discussed in the context of the future of plant virology.

\section{EUPHRESCO: As part of a network of research endeavors towards global surveillance}

B. GIOVANI, European and Mediterranean Plant Protection Organization-Euphresco, Paris, FRANCE

The Euphresco network for phytosanitary research coordination and funding was initiated as an EU-funded project in 2006 with the aim to increase cooperation and coordination of national phytosanitary research programmes through networking of research activities and mutual opening of national programmes. Coordination allows to optimise research provisions that underpins quarantine plant health policy development and policy implementation, in an era of increasing biosecurity threats and increase the capacity of European phytosanitary science and research, in order to prevent the disappearance of expertise in this field. Since 2014, the network is hosted within the European and Mediterranean Plant Protection Organization (EPPO) and has evolved into a self-sustainable network supported by its members and accountable to them. In a globalised world, plant health challenges can no longer be seen from a national or regional point of view. A comprehensive approach is needed to facilitate the involvement of plant health operators and stakeholders to create more diverse and critical mass and to allow more efficient use of national resources. Reducing fragmentation also favours capacity building, knowledge sharing and harmonisation of methods. The Euphresco network has succeeded to bring together research programme owners, programme managers, National Plant Protection Organizations (NPPOs), regulators, policy makers and research organizations from five continents. Every year, Euphresco members initiate small-medium size research projects that provide scientific evidence to support the operations of National Plant Protection Organizations and policy development. More than 100 projects (full list available at https://www.euphresco.net/projects/portfolio) have been initiated that cover activities such as surveillance, diagnostics, pest-risk analysis, pest management. The presentation will explain Euphresco, will provide a vision on global phytosanitary research coordination and will showcase examples of research projects that have contributed to enhance surveillance activities.

\section{The contribution of population heterogeneity to plant colonization} P. KANDEL (1), H. Ehau-Taumaunu (1), K. Hockett (2), (1) The Pennsylvania State University, University Park, PA, USA; (2) The Pennsylvania State University, University Park, PA, USA

Pseudomonas syringae is a Gram-negative, plant pathogenic bacterium that infects crops such as common bean, soybean, tomato, kiwifruit, and apple. For sustained infection, the bacterium has to withstand factors such as the host defense, competition, and environmental stressors. Strains of Pseudomonas syringae are known to produce bacteriocins, antibacterial protein toxins, that kill competitor strains occupying the same habitat. Here, by co-infiltrating bacteriocin producing and target populations, we demonstrate that the producing strain maintains a subpopulation of bacteriocin induced cells that sacrifice themselves to release bacteriocin. The bacteriocin antagonize the competitors and enable the producing strain to have a greater influence over the niche. We also show that the target strain maintains a persister-like subpopulation that survives bacteriocin without carrying fitness compromising mutations. Also, the target strain maintained persister cells against common antibiotics applied against it. Interestingly, the bacteriocin and antibiotics persisters were different as a combination treatment killed both. Taken together, plant pathogenic bacteria can maintain heterogenous phenotypic variants of the homogenous genetic population. These variants enable dominance against competitors or survival against stresses leading to sustained plant infection. The heterogeneity likely influences the effectiveness of both chemical and biological control measures.

\section{Pros and cons of different air samplers and spore identification methods} J. WEST, Rothamsted Research, Hertfordshire, ENGLAND

It is important to consider the purpose of sampling for airborne pathogens before deciding which sampler to use, where to locate it and what identification method to use. The usefulness of information obtained will depend on the volume of air sampled, sampling efficiency for the size of target particle, height of sampler above ground or proximity to crops, speed of obtaining results and ability to network multiple devices. This can help (a) to research the biology and epidemiology of a pathogen, (b) for practical disease forecasting / surveillance or (c) for monitoring changes in traits of a pathogen population. For use (a), samplers can be placed close to known sources of inoculum and a range of diagnostic methods are available, which can be lab-based. For (b) there are advantages of integrating sampling with rapid diagnosis and standardisation of sampling methods for comparison of different locations, for (c) usually DNAbased analysis is best but this may only be needed at one or a few snapshots in time when the target organism is abundant. Sampling issues revolve around volume of air sampled, collection efficiency for small or large targets, and effect of proximity to sources on the concentration of spores per litre of air sampled. Diagnostic or identification issues revolve around time to get results and similarity of spores of different species for visual or optical methods (including image analysis and automation based on light transmission or reflectance, fluorescence, size, texture, etc), or inhibition, cross-reactivity, and sensitivity for immunological and DNA-based methods.

\section{Dickeya dadantii differentiates into two subpopulations to multitask on virulence and growth during the infection of potato} Z. Cui, Q. ZENG, The Connecticut Agricultural Experiment Station, New Haven, CT, USA

Necrotrophic plant pathogens acquire nutrients from dead plant cells, which requires the disintegration of the plant cell wall and tissue structures by the pathogen. Infected plants lose tissue integrity and functional immunity as a result, exposing the nutrient rich, decayed 
tissues to the environment. One challenge for the necrotrophs to successfully cause secondary infection (infection spread from an initially infected plant to the nearby uninfected plants) is to effectively utilize nutrients released from hosts towards building up a large population before other saprophytes come. In this study, we observed that the necrotrophic pathogen Dickeya dadantii exhibited heterogeneity in bacterial cell length in an isogenic population during infection of potato tuber. While some cells were regular rod-shape $(<10 \mu \mathrm{m})$, the rest elongated into filamentous cells $(>10 \mu \mathrm{m})$. Short cells expressed all necessary virulence factors and motility, whereas filamentous cells did not engage in virulence, were non-mobile and more sensitive to environmental stress. However, compared to the short cells, the filamentous cells displayed upregulated metabolic genes and increased growth, which may benefit the pathogens to build up a large population necessary for the secondary infection. The segregation of the two subpopulations was dependent on differential production of the alarmone guanosine tetraphosphate ( $\mathrm{ppGpp}$ ). When exposed to fresh tuber tissues or freestanding water, filamentous cells quickly transformed to short virulent cells. The pathogen adaptation of cell length heterogeneity identified in this study presents a model for how some necrotrophs balance virulence and vegetative growth to maximize fitness during infection.

Temperature impacts on the effectiveness of the rice blast resistance gene Ptr Y. JIA (1), H. Box (2), X. Wang (3), H. Zhao (4), G. L. Wang (5), (1) USDA ARS DBNRRC, Stuttgart, AR, USA; (2) USDA ARS Dale Bumpers National Rice Research Center, Stuttgart, AR, USA; (3) UA/USDA, Stuttgart, AR, USA; (4) USDA ARS, 130, AR, USA; (5) The Ohio State University, Columbus, OH, USA

Knowledge on the impact of temperature on the effectiveness of defense responses to the rice fungal pathogen Magnaporthe oryzae that causes rice blast disease is largely unknown. The Ptr gene provides broad-spectrum resistance to most of the predominant rice blast races in the US, except for IE1k. Ptr is predicted to encode two protein isomers with 4 armadillo repeats and different amounts of the Ptr protein that occur between the nucleus and cytoplasm of the plant cell are critical for effective disease resistance. Rapid accumulation of transcripts of the Ptr gene after cold and heat treatments of rice germplasm were observed by other investigators. In the present study, we investigated the disease reactions of rice germplasm containing the Ptr gene treated with low temperature before infection by the blast pathogen $M$. oryzae. Seedlings at the 3-4 leaf stage of three Ptr containing rice germplasm lines, S/C272, S/C324, $\mathrm{S} / \mathrm{C} 353$, were subjected to $4^{\circ} \mathrm{C}$ for $4 \mathrm{hrs}$. The controls were the same germplasm without temperature treatment. Both treated and control germplasm were inoculated with IE1k. Approximately one day delayed disease development was observed after $4^{\circ} \mathrm{C}$ treatment in comparison with untreated controls. This result suggests that enhanced resistance to IE1k occurred after seedlings were exposed to $4^{\circ} \mathrm{C}$. Progress on disease reaction of rice seedlings with $42^{\circ} \mathrm{C}$ treatment will be presented.

\section{Strengthening the Global Regulatory Sector through Scientific collaboration} M. K. NAKHLA, USDA-APHIS-PPQ-S\&T, Beltsville Laboratory, Beltsville, MD, USA

The United States Plant Protection and Quarantine (PPQ) safeguards agriculture and natural resources from the risks associated with damaging plant pests to ensure an abundant, high-quality, and varied food supply. Science and Technology (S\&T) provides scientific support for PPQ regulatory and operational decision making and ensures that PPQ has the information, technology, tools, and methods needed for effective pest exclusion, detection, and management. At S\&T we established strong national and international collaboration to better safeguard agriculture and facilitate safe agricultural trade. The goal is to support regulations and regulatory decisions with best scientific evidence to protect US agriculture products from exotic pests while facilitating safe agricultural trade. New advanced surveillance, detection and identification technologies are evaluated and implemented. Global scientific collaboration is essential for globally accepted regulations to reduce losses of agriculture products due to damaging pests and to facilitate safe trade to reduce the introduction, spread and establishing of exotic pests. Timely exchange of information, application of strong quality assurance and management systems and implementing validated methods and protocols are important for global effective regulations.

An oomycete RXLR effector triggers antagonistic plant hormone crosstalk to suppress host immunity R. Anderson (1), D. Deb (1), J. Withers (2), S. Y. He (2), J. M. MCDOWELL (1), (1) Virginia Tech, Blacksburg, VA, USA; (2) Michigan State University, East Lansing, MI, USA

Understanding the mechanisms through which pathogens alter plant cell networks is essential for understanding plant-pathogen interactions and will inform efforts to reduce crop diseases. Oomycetes secrete diverse effector proteins into plant cells. The mechanisms through which these effectors promote virulence are largely unknown. We show that the HaRxL10 effector protein from the Arabidopsis thaliana pathogen Hyaloperonospora arabidopsidis (Hpa) targets a transcriptional repressor (JAZ3) involved in jasmonic acid (JA) signaling. This manipulation activates a regulatory cascade that inhibits salicylic acid (SA) signaling, which normally restricts $\mathrm{Hpa}$ infection. This virulence mechanism is functionally equivalent to but mechanistically distinct from activation of the antagonistic JA-SA hormone crosstalk by the bacterial JA-mimicking toxin coronatine and by bacterial Type III effectors. These results reveal a key role for JAZ3 in plant immunity and emphasize that JA-SA crosstalk is an Achilles' heel in the plant immune system, vulnerable to manipulation by diverse microbes.

Exploring the global regulatory network of cyclic-di-GMP in Erwinia amylovora R. R. KHARADI, Michigan State University, East Lansing, MI, USA

Erwinia amylovora is the causal agent of fire blight, an economically impactful disease of apple and pear. An E. amylovora infection consists of alternating type III secretion dependent and biofilm dependent phases. Cyclic-di-GMP (c-di-GMP) is a critical second messenger that regulates the transition between these phases of infection. Here, we aimed to investigate the global regulatory effect of cdi-GMP and to dissect the evolutionarily critical role of each individual enzyme involved in c-di-GMP synthesis (diguanylate cycles 
$e d c A-E$ ), degradation (phosphodiesterases $p d e A-C$ ), as well as four other proteins with degenerate GGDEF/EAL domains in $E$.

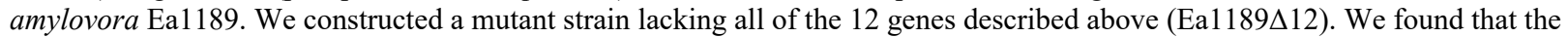
Ea1189 12 strain was unable to attach to a surface, and this impaired its ability to form biofilms in a flow-based system. RNA-Seq analysis of surface treated Ea1189 12 vs. wild type cells revealed globally contrasting transcriptional patterns within the two conditions. Chromosomally complementing Ea1189 12 with each of the deleted genes revealed that each diguanylate cyclase and phosphodiesterase enzyme confers unique regulatory effects on amylovoran production, modulation of the type III secretion system,

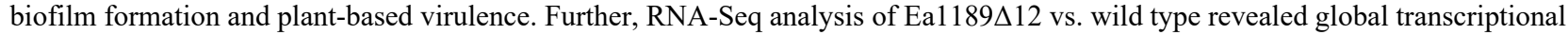
differences, particularly with differential gene set enrichment in important categories like pathogenesis, exopolysaccharide production and several metabolic pathways.

\title{
Pathogen synergism of blackleg disease on potato
}

T. GE (1), S. Johnson (2), R. P. Larkin (3), A. O. Charkowski (4), J. Hao (1), (1) School of Food and Agriculture, University of Maine, Orono, ME, USA; (2) University of Maine Cooperative Extension, Presque Isle, ME, USA; (3) USDA-ARS, New England Plant, Soil, and Water Lab, Orono, ME, USA; (4) Colorado State University, Fort Collins, CO, USA

Blackleg and soft rot of potato can be caused by individual species or a complex of Dickeya and Pectobacterium spp. Potato samples with blackleg and soft rot symptoms showed that a third of them were co-infected by both $D$. dianthicola and $P$. parmentieri. In addition, many samples contained even more complex assemblages of causative organisms, including $P$. brasiliensis, $P$. oderiferium, and $P$. polaris. Among these species existing in symptomatic potato tissues, $D$. dianthicola and $P$. parmentieri were tested to have greater pathogenicity than the others, and therefore were used in further investigations. To determine the role of these two bacterial species on disease expression, a field trial was conducted in Maine. Seed pieces of potato 'Shepody', 'Lamoka', and 'Atlantic' were inoculated with $D$. dianthicola, $P$. parmentieri, or mixture of both species using a vacuum infiltration method. The tubers were planted in a randomized block designed field trial. Blackleg symptoms appeared 6 weeks post planting. Potato inoculated with $D$. dianthicola had higher disease incidence (16.22\%) than with P. parmentieri $(10.4 \%)$, but the highest disease incidence was observed when the two species were co-inoculated $(25.43 \%)$. This result was consistent across all three potato varieties in two-year trials. Therefore we proposed, co-infection of two bacterial species synergistically promoted disease development at a higher level.

\section{The kaleidoscopic future of plant virology} D. MOLLOV, USDA, Beltsville, MD, USA

Plant viruses and the diseases they cause have been an agricultural problem for millennia and continue to provide some of our greatest challenges in food, fiber, and floral production. Plant virologists need to adopt new and multi-faceted approaches and to constantly evolve. Great scientific discoveries and applications in agricultural systems enable adequate responses to continuously growing societal changes and challenges. However, new changes and challenges always occur and rapid interventions in agricultural and natural systems will always be necessary. There is a rich history of breakthroughs and advances in plant virology. Forward thinking technological innovations are only possible when built on existing knowledge and methodology. Currently, genomic-based approaches, like deep sequencing, can be merged with proteomics, imaging, and other powerful technologies to develop robust discovery pipelines for new viruses. Further emphases on viral genome plasticity, host genomes, resistance genes, virus-vector interactions, vector movements, and population and epidemiological studies as part of complex biological networks would produce new insights enabling plant virologists to meet emerging challenges and agricultural threats. This integrated methodology, coupled with an open-minded approach, can meet the challenge of the exciting world of existing and emerging viruses which continuously threaten food production and human well-being.

\section{The US National Plant Diagnostic Network (Panel discussion) C. LAPAIRE HARMON, National Plant Diagnostic Network, FL, USA Univ of Florida PDC, Gainesville, FL, USA}

The National Plant Diagnostic Network (NPDN) is a consortium of plant diagnostic laboratories built on the US land-grant-university extension system. At least one plant diagnostic lab serves every state and most territories (72 active labs as of 2019), serving their local clientele and national surge and surveillance partners. Established by USDA in 2002 in response to biosecurity concerns post-9-11, the NPDN comprises five regions based on crop and climate, as well as a national data repository (based at Purdue University). The main emphasis of these labs tends towards plant disease diagnosis, but includes expertise in entomology, nematology, and weed science. Additionally, partners including APHIS, state departments of agriculture, extension, and IPM, compound the contacts and effectiveness of the network. As of 2020, the NPDN is in the implementation stage of a new strategic plan that focuses efforts on diagnostics, professional development, communications, and the IT systems that underpin the whole network. With renewed focus on diagnostic method development, implementation, and validation, NPDN is looking to its national and international partners to ensure broad surveillance and effective detection methods are in place for 2020 and beyond.

\author{
Integrating global databases for risk analysis \\ K. A. GARRETT, Plant Pathology Department, University of Florida, Gainesville, FL, USA \\ Institute for Sustainable Food Systems, University of Florida, Gainesville, FL, USA
}

Smart, data-driven surveillance systems are necessary to reduce the risk of pathogen establishment and threats to food security. As illustrated dramatically by both human and crop pandemics, an agile early response is key for effective mitigation. If data known to be relevant to epidemic risk are integrated in advance, the response can be quicker. Important categories of geographic data layers for 
interpreting epidemic risk include climate, recent weather, cropland connectivity, trade networks, farmer management practices, and regional and national phytosanitary practices. The other critical component is pathogen tracking from data collection by farmers, national and international programs, and agribusinesses, through plant sample testing and image analysis. Smart surveillance systems employ disease forecasting models for local disease severity and epidemic spread across regions. Models identify target locations and timing for surveillance and mitigation, and the most important types of new data to reduce uncertainty. A global surveillance system (GSS) for plant disease can address the challenges and technical demands of smart surveillance. Building a community of practice for disease risk assessment can support global capacity for applying and interpreting models and deploying them to fight pathogen invasions. By integrating current data layers and models, GSS teams can have effective strategies ready when emerging pathogens arise.

The role of plant hormones in cuticle formation and related function against the bacterial pathogen infection Y. XIA, Z. Zhao, J. Fan, Y. Liang, P. Yang, H. Kwok, The Ohio State University, Columbus, OH, USA

Plant cuticle is the outermost layer of a plant that interacts with environment and protects plants from water loss and various biotic and abiotic stresses. We firstly identified that a key component of the vesicle trafficking system, which encodes one member of adenosine diphosphate (ADP) ribosylation factor (ARF) guanine nucleotide exchange factor (GEF) protein family of Arabidopsis plants, is critical for the cuticle formation and related defense against the pathogen infection. Plant hormones have been reported to play roles in plant cuticle formation. By the exogenous application of multiple plant hormones, our study showed that SA (Salicylic Acid), JA (Jasmonic Acid), and ABA (Abscisic Acid) could specifically rescue the cuticle formation in the cuticle-defective leaves of the mutant plants. And the enhanced cuticle formation was related to the increased defense responses against the bacterium Pseudomonas syringae infection. Meanwhile, the UPLC analysis showed that the SA, JA and ABA levels in the leaves of mutant plants were significantly decreased compared to the wild type Col-0 plants. The levels of multiple marker genes of related hormone pathways were also down regulated in the mutant plants compared to wild type plants. Thus, we discover that this vesicle trafficking related component was critical for the associations between plant hormones and plant cuticle formation in relation to the defense responses against the bacterial pathogen infection. Our further study on the detailed mechanisms and related networks may facilitate the development of efficient strategies to enhance plant health and yield through the genetic manipulation of the plant cuticle.

\section{Adaptation of Xylella fastidiosa to new hosts and spittlebug vectors: An emerging threat to olives and other Mediterranean crops M. SAPONARI, CNR - IPSP Italian National Research Institute for Sustainable Plant Protection, Bari, ITALY}

Recent epidemics of Xylella fastidiosa in the European Union (EU), specifically in some Mediterranean countries, highlights the detrimental impact caused by establishment and spread of an invasive pathogen into new regions that results in a vast crop destruction before effective mitigation can be developed and/or implemented. Historically, X. fastidiosa has been confined to the Americas, causing devastating yield losses in grapes and citrus but also infecting a wide range of host plants asymptomatically. In the EU, a Xylellaepidemic emerged in southern Italy in 2013 and spread rapidly decimating olive trees with a disease called Olive Quick Decline Syndrome (OQDS). Investigations showed that this bacterium was introduced with infected plants from Central America and OQSD was caused by an exotic strain identified as $X$. fastidiosa subspecies pauca, sequence type 53 . The high susceptibility of olive to this pathovar along with the abundance of competent insect vectors, principally, indigenous spittlebug species and favorable environmental conditions, has resulted in destruction of the olive industry in this Italian olive-growing region. The prevalence of OQDS makes it one of the most severe epidemics associated with X. fastidiosa worldwide. Other European countries (France, Spain, Portugal) have now reported Xylella-outbreaks, albeit, to a lesser extent, in different species of the Mediterranean flora. In the last 5 years, EU research programs significantly advanced the knowledge on the introduction of $X$. fastidiosa in Europe and future perspectives for its control: (i) adaptation of the bacterium (with the multitude of strains so far detected in Europe) to several plant species of the Mediterranean flora, never exposed to the bacterium before, increase the uncertainties on the outcomes of the infections and on the plant responses; (ii) in this regard, the infections on olives is an explicative example of a novel host-pathogen combination (strains of the subspecies pauca were never reported before in olives) turning in a severe unpredictable disease; (iii) several strains detected in Europe were never characterized before, indicating that there is still a large undisclosed genetic and biology diversity; (iv) the database of susceptible host plants of the bacterium increased significantly upon its finding in Europe suggesting the high vulnerability of the Mediterranean ecosystems; and (v) in contrast to common sharpshooter vectors of Xylella in the Americas, the predominant xylem-sap feeder spreading the bacterium in the EU are spittlebugs. Therefore, targeted studies are focused on understanding vector transmission behavior, dispersal patterns, and population dynamics to develop sustainable management strategies for this vector and associated Xylella diseases. Finally, investigations, based mostly on genetic data, carried out in the EU show different introductions of the bacterium likely occurred in the past in EU with the commercial trade of plant material. Hence, policies aimed at better detection, inspection, surveillance, enforcement and public education would reduce inadvertent exotic pathogen introduction to new regions.

High throughput ANI analysis reveals clear species boundaries K. KONSTANTINIDIS, Georgia Institute of Technology, Atlanta, GA, USA

The 16S rRNA (16S) gene has served effectively as the backbone of prokaryotic taxonomy but offers limited resolution at species and subspecies levels, cannot assess functional gene diversity, and is often not assembled as part of the metagenome-assembled genomes (MAGs) recovered from environmental DNA, limiting its usefulness. To address these limitations, we have developed the "genomeequivalent" of the 16S webservers, the Microbial Genomes Atlas (MiGA) project (http://microbial-genomes.org/). MiGA employs the Average Nucleotide and Amino Acid Identity (ANI/AAI) concepts, and the distribution of ANI/AAI values among type genomes to 
classify a query genome against the described genomic diversity and provide the confidence level for the classification. The computational speed is partly facilitated by FastANI, a $k$-mer-based ANI estimator (Jain, Nat. Comm. 2018). FastANI also allowed us to explore the distribution of ANI values among $>90$ thousand complete and draft genomes available as part of MiGA's reference database. This distribution revealed a pronounced valley in the ANI range $83-95 \%$, indicating that the genetic discontinuity previously observed with smaller sets of genomes, reflective of discrete species, is maintained in larger scales. Examples from applying these concepts and tools to identify the causative agents of foodborne diarrhea, including novel pathogens, will be presented.

\section{Regional management of Fusarium head blight in Brazil and Pennsylvania: Integrative risk assessment approaches using} literature and field survey data

M. R. DUFFECK (1,2), E. M. Del Ponte (3), P. Esker (2), (1) Departamento de Fitopatologia, Universidade Federal de Viçosa, Viçosa, BRAZIL; (2) The Pennsylvania State University, University Park, PA, USA; (3) Departamento de Fitopatologia, Universidade Federal de Viçosa, BRAZIL

Fusarium head blight (FHB) threatens small grain production in the Northeastern U.S. and in Brazilian subtropics and presents unique challenges for regional management of the disease. In Brazil, a systematic review was conducted to obtain FHB-yield data and explore the relationship with FHB using a meta-analytical approach. Studies were grouped into low $(Y l<3631 \mathrm{~kg}$ ha- 1$)$ or high $(Y h>3631 \mathrm{~kg}$ ha-1) yield environments. Population-average intercepts differed between $Y l(2,883.6 \mathrm{~kg}$ ha-1) and $Y h(4,419.5 \mathrm{~kg}$ ha-1), but slopes were similar. The calculated relative yield loss ( $D c$, damage coefficient) was $1.05 \%$ pp-1 and $1.60 \%$ pp-1 for $Y h$ and $Y l$, respectively. Additionally, yield losses were estimated over a 28-year period, and the probability of not-offsetting (Ploss) the costs of one or two sprays of tebuconazole was calculated. One spray with tebuconazole proved to be a more profitable strategy to manage FHB after the 1990s. In Pennsylvania, a total of 349 Fusarium spp. isolates were recovered from FHB-infected spikes of different hosts. We found that $94.5,4.5$, and $1 \%$ belonged to $15-\mathrm{ADON}, 3-\mathrm{ADON}$, and NIV genotypes, respectively. We also observed no known resistance of Fusarium graminearum $(\mathrm{Fg})$ isolates to tebuconazole and metconazole fungicides. Comparing isolates across three distinct management regions, there was no evidence of differences in terms of aggressiveness. Current work is focused on investigating the genetic diversity of a subset of Fg isolates using VNTR markers. New results from PA will inform and strengthen current FHB and mycotoxin management strategies.

Genomic variability and evolutionary relationships of badnaviruses infecting cacao J. K. BROWN, University of Arizona, Tucson, AZ, USA

In West Africa, cacao swollen shoot disease (CSSD) badnaviruses are economically important pathogens of the cocoa tree (Theobroma cacao L.) causing yield loss and tree death. Foliar symptoms develop on flush growth after the rainy seasons, and are relatively ephemeral as leaves mature. Swellings, the characteristic from which the disease name is derived, develop on roots, stem, and shoots. The disease is caused by a group of badnaviral species [genus, Badnavirus; family, Caulimoviridae]. The CSSD badnaviruses have a circular, gapped dsDNA genome, ranging in size from $\sim 6.8-7.3 \mathrm{kbp}$, encapsidated in a non-enveloped bacilliform particle $\sim 128 \times 28$ $\mathrm{nm}$. Phylogeographic (Bayesian, ML) analyses of CSSD-West African and other known cacao-infecting badnaviruses groups the west African viruses into 6-7 clades or putative species. The African-CSSD badnavirus isolates are most closely related to one another while isolates from the Caribbean region are thought to be endemic to the American Tropics. Pairwise distance analysis of the RT-RNase H region and of whole genome sequences, respectively, indicate $\sim 70-100 \%$ and $61-100 \%$ shared nt identity, and is consistent with ten species overall. Observations suggest that extant CSSD-associated badnaviral species causing swollen shoot disease share a common ancestor(s) and that extant isolates found in cacao have made independent host-jumps from the wild to cacao host, shortly after the introduction of cacao approximately 100 years ago. Even so, additional host jumps may also be contemporary events. Collectively, the analyses support a West African endemism for CSSD-badnavirus species, and implicate the western region of Africa as the center of diversification.

Anthropogenic influences on widespread decline of eastern white pine I. A. MUNCK (1), M. L. Sakalidis (2), (1) USDA Forest Service, Durham, NH, USA; (2) Michigan State University, East Lansing, MI, USA

Eastern white pine (Pinus strobus L.) is a critical ecological and economic component of forests in eastern North America. During the last century, the tree's abundance has been affected by logging, field abandonment, introduction of the non-native white pine blister rust and native white pine weevil. Within the last decade, eastern white pines have experienced unprecedented damage associated with previously innocuous native insect and pathogen species including Caliciopsis pinea Peck, Matsucoccus macrocicatrices Richards, and a complex of foliar pathogens generally referred to as White Pine Needle Damage (WPND). Climate change predictions forecast warmer and wetter climates for the Northeastern US in coming years. Increased precipitation in June has been linked to increased damage by foliar pathogens, whereas hotter summers and drought have been associated with increased tree stress and Caliciopis canker damage. This presentation will summarize the latest developments in eastern white pine health including elucidation of causal agents, damage quantification (such as economic loss), and management recommendations to reduce risk of future damage within the context of climate change.

Management of Fusarium oxysporum and Pythium spp., causing root and crown rot, and powdery mildew (Golovinomyces cichoracearum) on cannabis plants

C. SCOTT, Simon Fraser University, Burnaby, CANADA 
Crown rot, root rot and damping-off caused by Fusarium oxysporum and Pythium spp. reduce growth of cannabis plants. Infection may occur on cuttings and vegetative plants, and wounding of roots enhances infection. To evaluate the effectiveness of biological control agents for disease management on cuttings, Rhapsody ${ }^{\circledR}$ ASO, Stargus ${ }^{\circledR}$, Prestop ${ }^{\circledR}$, RootShield ${ }^{\mathbb{R}}$ Plus WP, Asperello ${ }^{\circledR}$ and Regalia ${ }^{\circledR}$ Maxx were applied $48 \mathrm{hr}$ prior to $F$. oxysporum inoculation. The most effective treatments were Regalia ${ }^{\circledR}$ Maxx, Rootshield ${ }^{\circledR}$ Plus WP, Stargus $^{\mathbb{B}}$ and Rhapsody ${ }^{\circledR}$ ASO. Root infection by Pythium was most effectively reduced by either Rootshield ${ }^{\mathbb{R}}$ Plus WP or Prestop ${ }^{\circledR}$, applied as a drench 1 week and $24 \mathrm{hr}$ prior to pathogen inoculation on rooted plants. Both treatments caused a significant $(\mathrm{P}<0.05)$ increase in shoot height and root length compared to the nontreated inoculated group. Powdery mildew on cannabis, caused by Golovinomyces cichoracearum, can cause a loss of vigor and yield, and in severe causes may infect the inflorescences. For powdery mildew management, treatments of Actinovate ${ }^{\circledR}$, MilStop ${ }^{\circledR}$, Regalia Maxx ${ }^{\circledR}$, Rhapsody ${ }^{\circledR}$ ASO, Stargus ${ }^{\circledR}$ and ZeroTol ${ }^{\circledR}$ were made

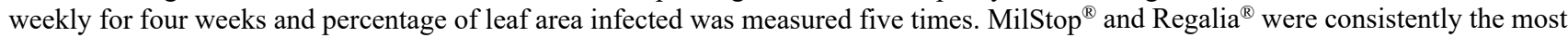
effective treatments and both provided significantly $(\mathrm{p}<0.05)$ lower AUDPC values compared to nontreated plants. Plants were also exposed daily for 3-5 sec to UV-C light from a CleanLight Pro unit to test the effects on mildew development. Results showed disease reduction up to $45.2 \%$.

Fungicide resistance: Screening and risk-assessment of Rhizoctonia zeae populations in Nebraska N. GAMBHIR (1), S. Kodati (2), A. O. Adesemoye (3), S. E. Everhart (1), (1) University of Nebraska, Lincoln, NE, USA; (2) Connecticut Agric Exp Sta, New Haven, CT, USA; (3) Terramera Inc, Vancouver, BC, CANADA

Fungicide seed treatments are commonly used to manage seedling diseases of corn and soybean. Knowledge of the fungicide resistance profile and risk of resistance development is relevant for managing fungicide resistance in Rhizoctonia zeae, the dominant Rhizoctonia species pathogenic to corn and soybean seedlings in NE. In vitro fungicide sensitivity was determined for $49 R$. zeae isolates that were obtained from corn and soybean fields in NE in 2016 and 2017. The average effective concentration of 50\% inhibition for the fungicides tested were: fludioxonil, $0.099 \mathrm{ppm}$; prothioconazole, $0.233 \mathrm{ppm}$; sedaxane, $0.079 \mathrm{ppm}$; and azoxystrobin, >100 ppm. Rhizoctonia zeae was sensitive to all fungicides except azoxystrobin. In planta assays with two $R$. zeae isolates showed that azoxystrobin treatment did not significantly change the disease severity index, stand count, or total weight of soybean plants $(P>0.05)$, suggesting that $R$. zeae is insensitive to azoxystrobin. Characterization of population structure will help to assess the risk of resistance development for this pathogen. For this, $43 \mathrm{SSR}$ primers were designed and evaluated on $13 \mathrm{R}$. zeae isolates. Preliminary results showed that seven primers produced consistent amplification and polymorphisms, with an average of 5.86 alleles, medium-high gene diversity $\left(H_{\exp }=0.72\right)$, and medium-high evenness $(E .5=0.78)$. These primers will be used to characterize the population structure of $106 R$. zeae isolates. Results will help to assess the risk of fungicide resistance development in $R$. zeae and design effective management strategies.

\section{Aphid-transmitted Cotton leafroll dwarf disease: An emerging threat to sustainable production of cotton}

S. BAG (1), A. Tabassum (1), N. Sedhain (1), R. Kemerait (1), P. Chee (1), J. Whitaker (1), N. D. Suassuna (2), R. Nichols (3), P. Roberts (1), (1) University of Georgia, Tifton, GA, USA; (2) EMBRAPA, Campina Grande, BRAZIL; (3) Cotton Incorporated, Cary, NC, USA

Cotton leafroll dwarf virus (CLRDV) is a single-stranded positive-sense, phloem restricted, RNA virus transmitted by cotton aphids in a persistent circulative manner and is the presumptive cause of cotton leafroll dwarf disease. First identified in 2018 in AL, CLRDV was identified subsequently in 2019 in all other major cotton-producing states in and east of Texas, except Oklahoma. Early disease symptoms include reddening and drooping of leaves and 360-degree reddening of petioles. As the disease progresses, symptoms may diminish but may be followed by more severe symptoms including leaf rigidity, crinkling, and abortion of fruiting forms resulting in yield loss. Symptoms are apparently influenced by geographical location, varieties, plant health, and cultural practices. In 2019 , the virus was detected from asymptomatic plants and numerous weeds. Based on the whole genome sequence, the isolates from GA shared a high level of homology with those reported from AL and TX in the U.S., and atypical strains of CLRDV reported earlier from South America. In 2018-19 the disease did not cause demonstrably significant yield loss, but the occurrence of stunted plants, observation of low boll retention, and discovery of egregious damage (estimated $20 \%$ yield loss) in isolated fields raises concerns about the future impact on cotton production.

\section{Importance of climate, anthropogenic disturbance and pathogens on marri tree health in Australia} T. PAAP (1,2), S. Sapsford (2,3), T. I. Burgess (2), G. E. Hardy (4), (1) Forestry \& Agricultural Biotechnology Institute, University of Pretoria, Pretoria, SOUTH AFRICA; (2) Environmental and Conservation Sciences, Murdoch University, Perth, AUSTRALIA; (3) School of Biological Sciences, University of Canterbury, Christchurch, NEW ZEALAND; (4) Environmental and Conservation Sciences, Murdoch University, Perth, Western Australia, AUSTRALIA

Corymbia calophylla (marri), is a keystone tree species of southwest Western Australia. In recent decades, susceptibility of this tree to cankers caused by the endemic fungus Quambalaria coyrecup, has emerged. An increase in the impact and severity of this pathogen has led to widespread tree decline and death. It poses an important question of why this pathogen, which has co-evolved with its host, has become so devastating. We used Manion's 'tree decline spiral' as a framework to examine the possible predisposing, inciting and contributing factors that could be linked to increased occurrence and severity of the canker disease. Studies were conducted at a coarse scale landscape level and finer scale forest stand level. We examined the role of anthropogenic pressures including fragmentation, pesticides and fertilizer inputs, changes in mycorrhizae and other soil microflora, and the role of a drying and warming climate on the incidence and severity of the disease. We found anthropogenic pressures to play a key role in the disease syndrome. Changes in soil properties, changes in mycorrhizal fungal communities, the presence of alien invasive Phytophthora species, and forest community composition and stand level structural traits were strongly associated with canker incidence and severity. We discuss these in detail. This study highlights the complexity of addressing tree health issues in the presence of multiple global change factors. 
Drought-influenced forest pathogen interactions of the western North America: A focus on Armillaria root disease J. STEWART (1), J. W. Hanna (2), B. M. Lalande (3), M. V. Warwell (2), M. S. Kim (4), N. B. Klopfenstein (2), (1) Colorado State University, Fort Collins, CO, USA; (2) USDA Forest Service, Rocky Mountain Research Station, Moscow, ID, USA; (3) Colorado State University-Agricultural Biology, Fort Collins, CO, USA; (4) USDA Forest Service, Corvallis, OR, USA

Drought conditions are associated with climate change in much of the western North America. As a result, physiological stress in trees is expected to rise due to maladaptation, and subsequently, the susceptibility to and impact of forest diseases is expected to increase. A predominate hypothesis is that increased risk of forest root diseases will be driven by forest pathogens that are indirectly affected by climatic changes and are associated with different woody tissues (e.g., root and canker diseases) and overall forest declines. Root diseases, such as those caused by Armillaria spp., are especially important in western forests as both precursors and consequences of tree stress. Root diseases are predicted to become more severe, and potentially expand into new geographic regions where climatic conditions exacerbate tree stress, but remain favorable for the pathogen. Such changes may result in increased growth loss and mortality of diverse tree hosts.

Orchards on Fire: Tracking fire blight between and within orchards using CRISPR strain characterization A. WALliS (1), P. Ramachandran (2), R. Timme (2), J. Pettengill (2), R. Cagle (3), A. Ottesen (2), K. D. Cox (1), (1) Cornell University, Geneva, NY, USA; (2) FDA, College Park, MD, USA; (3) University of Washington, Seattle, WA, USA

Tracking the distribution and movement of pathogen strains has the potential to dramatically increase understanding of disease epidemiology and improve management strategies, but requires the ability to efficiently identify strains from disease samples. Erwinia amylovora (Ea), causal agent of fire blight of apples, is a bacterial pathogen capable of rapid spread and destruction of large acreage in a single season over the course of only a few weeks. It may spread locally via weather events and insect vectors, or regionally on living plant tissue. Recently, sequencing and characterization of CRISPR regions in $E a$ has been used to differentiate and investigate genetic diversity of strains. Using this method, we identified predominant strains located in commercial apple production regions of NY State and examined distribution and spread within orchard blocks over multiple years. Whole genome sequence analysis of select strains in combination with CRISPR characterization elucidated regional clustering of strains and higher diversity in the Hudson Valley of NY, which is believed to be the center of origin of the pathogen. We also investigated ways to improve efficiency of the strain identification pipeline using multiplexed, high throughput sequencing. We envision developing an online platform to track $E a$ and other plant pathogens in real time, modeled after the GenomeTrakr system developed by NCBI and FDA for tracking human pathogens.

\section{Grapevine red blotch virus: Nearly a decade of progress in research and disease management} E. CIENIEWICZ, Clemson University, Clemson, SC, USA

Grapevine red blotch virus (GRBV) has likely been a persistent inhabitant of North American wine grapes for decades. Grapevine red blotch virus is a new member of the family Geminiviridae. GRBV has proven different from other geminivirids, and also quite different from other viruses of grapevine, in its biology and transmission. Although we are only beginning to understand GRBV transmission biology and genome expression strategies, major strides have been made in the areas of disease etiology, disease ecology, and the viticultural and enological impacts of GRBV. As nearly a decade of red blotch disease research has accumulated, we reflect on the progress made and the advancements to come.

Pathogen-mediated hormone imbalances determine the growth and defense responses N. DHAR (1), A. Anchieta (2), B. Day (3), C. Casteel (4), K. V. Subbarao (5), S. J. Klosterman (2), (1) University of California, Davis, Salinas, CA, USA; (2) USDA ARS, Salinas, CA, USA; (3) Michigan State University, East Lansing, MI, USA; (4) Cornell University, Ithaca, NY, USA; (5) University of California At Davis, Salinas, CA, USA

Hormones play a central role in cellular communications coordinating multiple plant processes, including development and stress responses. Hormone signaling thus contributes to the survival and fitness of the plant under normal growth conditions as well as under stress from biotic and abiotic factors. Studies of hormone signaling have previously established that salicylic acid (SA) and jasmonic acid are primarily defense-related hormones. Auxin, gibberellins (GA), and brassinosteroids are tightly linked with growth, while other hormones like abscisic acid, cytokinin, ethylene, strigolactones are associated with multiple aspects of plant health. Work on various pathosystems has provided insights into the crosstalk among the classically studied hormones, linking hormone signaling pathways and defense. Our work on the interaction between the hosts Arabidopsis and lettuce and the soil-borne pathogen Verticillium spp. has shown that changes in host hormone levels are impacted by and influence responses of hosts to infection. During the early phases of Verticillium spp. infection, wild type Arabidopsis showed accelerated growth in a GA-dependent manner. Arabidopsis plants with the ndr1-1 mutation in the Nonrace specific Disease Resistance (NDR1) gene and defective in SA-signaling are highly susceptible to Verticillium spp. and show GA-dependent accelerated development, suggestive of SA-GA crosstalk between plant defense and developmental pathways. Hormone imbalances were assessed in Verticillium-Arabidopsis interactions, and the significance of GA signaling in lettuce and other hosts in response to Verticillium spp. was further explored.

Climate and biotic agents in sudden aspen decline

J. J. WORRALL (1), S. Marchetti (2), (1) USDA FS, WA, USA; (2) USDA Forest Service, Gunnison, CO, USA

Sudden aspen decline followed the turn-of-the-century drought in western North America, but continued to expand and intensify for years after this record drought. No primary biotic agents were involved, but many secondary agents contributed to mortality. Cytospora 
canker, aspen bark beetles (ABB), and bronze poplar borer (BPB) were most important and were much more abundant in SAD stands than in healthy. On trees with the same levels of crown loss, ABB and BPB attacked much more in damaged stands than in healthy, indicating density-dependent success. Increased population density in damaged stands resulted in more aggressiveness. Cytospora canker, however, behaved the same with respect to crown loss in healthy and damaged stands. This indicates that, for Cytospora (which can be endophytic in aspen, with latent infections causing cankers after stress), inoculum is not a limiting factor, and instead it is strictly stress-related. Other insects and pathogens likely play roles like BPB and ABB in declines related to climate stress, where increased population density results in increased aggression and killing of unstressed trees. These high populations likely contributed to the continued spread and intensity of SAD long after drought stress abated.

Identification of sensor-based signatures of peanut infection with Athelia rolfsii using machine learning X. WEI (1,2), M. Aguilera (2,3), S. Li (2), D. B. Langston Jr. (1,2), H. L. Mehl (4), (1) Virginia Tech Tidewater AREC, Suffolk, VA, USA; (2) School of Plant and Environmental Sciences, Virginia Tech, Blacksburg, VA, USA; (3) Genetics, Bioinformatics, and Computational Biology program, Virginia Tech, Blacksburg, VA, USA; (4) USDA Agricultural Research Service, Tucson, AZ, USA

Southern stem rot (SSR), a soilborne disease caused by Athelia rolfsii, suppresses peanut yields worldwide. Efficient, accurate disease detection is critical to optimize management practices including timely fungicide applications. Sensors have been utilized to detect foliar diseases, but few studies have employed these technologies to detect soilborne diseases. Specific objectives of this study were to 1) characterize spectral and thermal responses of peanut to infection and colonization with $A$. rolfsii and 2) identify precise spectral signatures of SSR using machine learning. In a greenhouse experiment, lateral stems of peanut plants were either mock-inoculated or inoculated with $A$. rolfsii and inspected daily for symptoms. Leaf temperature and spectral reflectance were measured with a handheld thermal camera and spectrometer, respectively. Three days after onset of wilting symptoms, infected stems had greater leaf temperature and percent spectral reflectance compared to non-infected stems. Spectrums assigned to three classes based on visual symptoms (healthy, mild symptoms, severely diseased) were used to train a random forest classifier resulting in an accuracy of $80.7 \%$ and $83.9 \%$ with primary and first-order derivative spectrums, respectively. Using signatures identified in this study, it may be possible to mount sensors on UAVs and remotely detect SSR in peanut fields. This study serves as a proof-of-concept for sensor-based detection of soilborne diseases and provides a foundation for integrating new technologies into precision scouting and application of inputs.

\section{Historical concept of bacterial species from host of isolation to phenetic analysis to DNA hybridization} J. B. JONES, University of Florida, Gainesville, FL, USA

The species concept in bacterial taxonomy was based primarily on a few phenotypic tests as the main criteria for species designation. For plant pathogenic bacteria the host of isolation was considered a very important criterion. Bacterial taxonomy was therefore subjective. With the advent of numerical taxonomy and molecular techniques for assessing bacterial divergence bacterial taxonomy became more objective. By 1980 there was a contraction of species for many plant pathogenic bacteria. The Ad Hoc Committee on Reconciliation of Approaches to Bacterial Systematics in 1987 indicated a hierarchical taxonomy should involve one system with complete DNA sequence serving as the reference for determining phylogeny and that phylogeny should be the basis of taxonomy. Furthermore, species was the only taxonomic unit that could be defined based on phylogenetic analyses. Furthermore they stated that nomenclature should be in agreement with phylogenetic information. Their view was the phylogenetic definition of species generally would include strains with approximately $70 \%$ or greater DNA-DNA relatedness and with $5^{\circ} \mathrm{C}$ or less DT $\mathrm{m}$. Furthermore phenotypic characteristics should be in agreement with the phylogenetic definition. They recommended that new species should only be named if there were phenotypic differences from the other species in that genus. We will discuss changes in nomenclature that occurred in various plant pathogenic bacteria following the recommendations of the Ad Hoc Committee.

\section{Cytokinins promote plant resistance to pathogens with different lifestyles C. ARGUESO, Colorado State University, Fort Collins, CO, USA}

Cytokinins are a group of plant hormones known for regulating plant growth and development. While first identified by their role in cell division, in the last decade these plant hormones have also been demonstrated to have a role in plant tolerance to abiotic, as well as biotic stress. With regards to biotic stress, application of high levels of cytokinins has been shown to reduce plant susceptibility to bacterial, oomycete and fungal pathogens, and is effective against pathogens of many lifestyles. The mechanism by which this plant growth hormone leads to reduced susceptibility to pathogens is still unknown, but lack of direct activation of defense indicates a mechanism similar to defense priming. We will present the most recent advances in understanding cytokinin-induced defense priming, and its practical uses in agriculture.

Cannabis and hemp biology and pathology: An overview of the crops and emerging pathogens Z. K. PUNJA, Simon Fraser Univ, Burnaby, BC, CANADA

Cannabis (Cannabis sativa L., marijuana) is grown indoors in controlled environments and in greenhouses, while hemp is grown outdoors. These plants are harvested for their inflorescences that produce various ratios of cannabinoids in trichomes, primarily THC and CBD. Plant pathogens infecting roots and crown tissues include Fusarium oxysporum, F. solani, F. proliferatum, $F$. brachygibbosum, $F$. graminearum, $F$. tricinctum, Cylindrocarpon lichenicola, Pythium dissotocum, $P$. myriotylum, $P$. ultimum, $P$. aphanidermatum and $P$. catenulatum. Symptoms include root browning, discoloration of crown and pith tissues, stunting and yellowing, 
and occasionally, plant death. On foliage, powdery mildew, caused by Golovinomyces cichoracearum, occurs in indoor and outdoor production, and a number of leaf spotting fungi have been reported on hemp. Recently, the hops powdery mildew pathogen, Podosphaeria macularis, was observed on field-grown cannabis plants. On inflorescences, botrytis bud $\operatorname{rot}(B$. cinerea, $B$. pseudocinerea), penicillium bud rot (caused by P. olsonii, P. copticola and other species), and fusarium bud rot ( $F$. solani, $F$. oxysporum, $F$. proliferatum, $F$. sporotrichiodes) occur to varying extents, depending on the environment. Hop latent viroid also infects the inflorescences. Approaches to disease management include application of biological control agents, sanitation methods, and selecting disease resistant genotypes. Future research avenues include the identification and cloning of resistance genes, development of tissue culture and transformation methods, and pathogen diagnostics.

\section{Recent advances in the epidemiology of hop powdery mildew: Characterizing aspects of early season ascosporic infection and pathogen dispersal \\ W. A. WELDON (1), K. D’Arcangelo (2), L. M. Quesada-Ocampo (3), M. E. Marks (4), A. J. Gevens (5), B. J. Knaus (6), N. J.} Grunwald (7), M. H. Block (8), D. Gent (9), L. E. Cadle-Davidson (10), D. M. Gadoury (1), (1) Cornell University, Geneva, NY, USA; (2) North Carolina State University, Raleigh, NC, USA; (3) Department of Entomology and Plant Pathology, North Carolina State University, Raleigh, NC, USA; (4) USDA-APHIS, Beltsville, MD, USA; (5) University of Wisconsin-Madison, Madison, WI, USA; (6) Department of Botany and Plant Pathology, Oregon State University, Corvallis, OR, USA; (7) USDA ARS, Corvallis, OR, USA; (8) Oregon State University, Corvallis, OR, USA; (9) USDA-ARS, Forage Seed and Cereal Research Unit, Corvallis, OR, USA; (10) USDA Grape Genetics Research Unit, Geneva, NY, USA

Hop powdery mildew (Podosphaera macularis) threatens sustainable hop production throughout much of the United States (US). $P$. macularis populations of the Pacific Northwest (PNW) differ greatly from those of the Midwest and Eastern US with respect to fungal virulence and modes of overwintering. The PNW currently harbors two pathogenic races that are virulent on cultivars with resistance based on the gene R6 and the cultivar Cascade. Further, only the MAT1-1 mating type idiomorph is known to occur in the region. Thus, unlike in most states east of the Rocky Mountains, the ascigerous state has not yet been observed in the PNW. The likelihood of eventual transfer of these unique $P$. macularis races and mating types among hop growing regions compelled us to (1) elucidate the role of ascocarps in the epidemiology of HPM and (2) develop a high-throughput genotyping tool to track pathogen movement. An initial project characterized early season re-emergence of $P$. macularis due to ascosporic infection alone, peaking at $70 \%$ disease incidence within the first month of bud break. Over consecutive seasons, $P$. macularis ascospore release was tracked across three geographies with diverse winter weather (NC, NY, and WI). When normalized by growing degree days accumulated since the date of first ascospore release, release curves fit to a beta regression model provided excellent estimates of the ascosporic infection period, irrespective of geographic origin. In a parallel study, we modeled the interaction of temperature and the duration of wetting events, fitting these variables to both a generalized linear mixed model and a modified Weibull distribution to estimate the degree of subsequent $P$. macularis ascospore release. Lastly, taking a population genetics approach, we re-purposed an existing, highly diverse $P$. macularis transcriptome dataset to create an amplicon sequencing SNP marker library capable of proactively monitoring the movement of unique and virulent strains throughout the US. These novel tools enable tracking of pathogen movement and forecasting the timing and intensity of early season ascosporic infection periods, which are critical components of IPM programs across many polycyclic fungal pathogens.

\section{Whole genome sequencing of plant pathogenic bacteria}

N. POTNIS (1), S. Timilsina (2), J. B. Jones (2), (1) Department of Entomology and Plant Pathology Auburn University, Auburn, AL, USA; (2) University of Florida, Gainesville, FL, USA

Whole-genome sequencing of multiple isolates belonging to each species as well as diverse species belonging to a single genus has accelerated our understanding of the genomic fluidity across closely related species. This genomic fluidity as result of rampant horizontal gene transfer events raises questions about the existence of the bacterial species. Conventionally species have been recognized to contain isolates that share complex phenotypic or biochemical features. Whole genome comparisons have allowed to revisit this species definition using concepts of average nucleotide identity (ANI) based on BLAST+ (ANIb) and MUMmer (ANIm), correlation indices of tetra-nucleotide signatures (Tetra) and most recently, core genome hypothesis. Core genome hypothesis posits that there is core gene set that encodes phenotypic properties that are signature of a species. In this presentation, we discuss all abovementioned approaches that have been used in the recent years for prokaryotic species circumscription. We also discuss specific examples of plant pathogenic bacterial species that have recently undergone reclassification.

\footnotetext{
Management thresholds: Finding and using them N. MCROBERTS (1), K. Coleman (2), R. Choudhury (3), G. Hughes (4), (1) University of California, Davis, CA, USA; (2) University of California, Davis, Davis, CA, USA; (3) University of Texas Rio Grande Valley, Edinburg, TX, USA; (4) SRUC, Edinburgh, UNITED KINGDOM

We address two issues connected with the use of thresholds in plant disease management. First, we review typical approaches for identifying and evaluating thresholds, focusing on situations where a threshold, either on early disease intensity assessments or a suitable risk variable, is used to differentiate crops that do require intervention from those that do not. Having established the methods by which thresholds might be found and used, our second topic focuses on potential problems that may arise from the long term use of thresholds in systems where there is inter-seasonal correlation in the intensity of disease. Using simple stochastic difference equations to represent disease dynamics we illustrate how imposing thresholds on noisy systems may lead to counter-intuitive effects on the long term frequency of action being taken and on the variability of disease over time.
} 
Botrytis cinerea, causal agent of gray mold, is a major pathogen of raspberry and other small fruit (berry) crops worldwide. $B$. cinerea can infect more than 2000 different plant species and is thought to be a generalist pathogen with little or no host specificity. Despite this ability to infect many different hosts, some studies have demonstrated that populations of $B$. cinerea are specialized to particular hosts. Other studies have failed to support host specialization and it remains to be determined if populations of this fungus are specialized to berry hosts. The objective of this study was to test the hypothesis that $B$. cinerea is a broad host range plant pathogen with no specialization to berry hosts by determining the genetic structure of $B$. cinerea populations infecting berries in the Pacific Northwest (PNW). Twenty single-conidial isolates of $B$. cinerea were sampled from each of 34 fields of red raspberry, blueberry, blackberry, wild blackberry, grapes, and currants on 11 farms in WA and OR, USA. A total of 663 isolates were collected and genotyped using 7 neutral microsatellite markers. Additionally, all WA isolates were screened for resistance to the succinate dehydrogenase (SDH) inhibiting fungicide boscalid using an in vitro assay. Sensitive and resistant isolates were further screened for resistance mutations in subunit B of SDH enzyme complex with PCR assays. Population genetic analysis of the microsatellite markers revealed high genetic diversity within populations with no evidence of recombination. STRUCTURE and DAPC analyses assigned all sampled populations to 2 and 7 genetic groups, respectively. No evidence of host specificity was observed. Frequencies of boscalid resistance differed among fields on the same farm ranging between 0 and $91.67 \%$ of total sampled isolates per field. Among resistant isolates, two types of amino acid substitutions (H272R and H272Y) were observed in codon 272 of subunit B of the SDH enzyme complex. The distribution of resistance alleles differed among fields from the same farm and there was no association of resistance allele with either host species, geography or farm. Both alleles were present in 18 fields, while only one allele was detected in 9 fields. The presence of private microsatellite and fungicide resistance alleles in adjacent fields indicated that $B$. cinerea populations in the PNW are highly localized with limited migration between them. AMOVA indicated that populations were significantly differentiated by geography. Significant pairwise differentiation was also detected among some adjacent fields on a farm scale providing further evidence of limited movement of $B$. cinerea in space. Moreover, significant negative correlations were detected between boscalid resistance frequencies and genic and genotypic diversities, while resistance frequencies were positively correlated with a clonal fraction. These results suggest that selection by fungicide use has a significant effect on pathogen diversity and has a stronger effect on population structure than selection by host. Our findings provide a better understanding of the population biology of the most important small fruit pathogen in the PNW and worldwide and will be used to improve gray mold management strategies by developing customized resistance management programs for individual fields based on fungicide resistance profiles in the PNW and elsewhere.

\section{Fungicide efficacy on target spot in Tennessee soybean and cotton}

T. SMITH (1), H. M. Kelly (2), Z. R. Hansen (3), (1) University of Tennessee, Jackson, TN, USA; (2) West Tennessee AgResearch and Education Center, University of Tennessee, Jackson, TN, USA; (3) University of Tennessee, Knoxville, TN, USA

Target spot (TS), caused by the fungus Corynespora cassiicola, is a foliar disease of cotton and soybean. Over recent years, TS has become a disease of concern in soybean production systems. Data for fungicide sensitivity and understanding potential impact on yield is lacking for C. cassiicola. The objective of this study was to conduct fungicide screening to monitor sensitivity in $C$. cassiicola in Tennessee soybean production. The sensitivity of $30 \mathrm{C}$. cassiicola isolates to 8 technical grade fungicides across multiple fungicide groups (FRAC Groups 1, 3, 7, and 11) was evaluated based on mycelial growth inhibition assays. The EC 50 of each fungicide was calculated. Field trials were also conducted at 3 locations for soybean in 2018 and 2019. Five fungicide tank mixes were evaluated for control of TS among 3 soybean varieties of differing susceptibility and 6 fungicide products were evaluated among 1 cotton cultivar. TS in soybean was decreased by all products except Domark, but only Miravis TOP protected yield in both years. Pyraclostrobin, thiophanate methyl, and azoxystrobin had the highest $\mathrm{EC}_{50}$ values. In vitro and field evaluations suggest that products that contain FRAC groups 7 and 3 have the potential to better protect yield from target spot compared to products containing groups 11 or 1 fungicides.

\section{Polyphasic approach to naming a bacterial species}

S. TIMILSINA (1), G. E. Vallad (2), J. B. Jones (1), (1) University of Florida, Gainesville, FL, USA; (2) Gulf Coast Research and Education Center, University of Florida, Wimauma, FL, USA

Bacterial nomenclature is regulated by the International Code of Nomenclature of Prokaryotes. However, the taxonomic classification to the species is not regulated. Bacterial taxonomy has been dynamic and regularly updated along with the technological advancements. Previously, DNA-DNA hybridization was regularly used for classification but has now shifted towards using the whole genome sequences (WGS). Various polyphasic approaches are used simultaneously when describing bacterial species for nomenclature and diagnostics. We will use several examples including plant pathogenic Pseudomonas spp., which are generally characterized by their fluorescent activity in iron deficient media followed by the use of standard LOPAT tests, to identify a new species. The inexplicable results in levan production by a group of Pseudomonas strains led to more in depth analyses that in turn led to the designation of the new species, $P$. floridensis. In addition to WGS, P. floridensis strains were compared to closely related Pseudomonas spp. using cellular fatty acid composition. The strains were subjected to Biolog assay characterizing species reaction to various growing conditions. Interestingly, the strains of $P$. floridensis seemed to react with sucrose during the Levan assay but showed no reaction with sucrose in the Biolog assay. The strains were later confirmed not to utilize sucrose, by plating in Aeyrs minimum media with $1 \%$ sucrose. In conclusion, polyphasic approaches are necessary to determine the characteristics and provide a meaningful taxonomy of novel species. 
High value, high risk-management thresholds in fresh fruits

N. PERES, Gulf Coast Research and Education Center; University of Florida, Wimauma, FL, USA

Strawberry is a high-value crop affected by several major diseases. Anthracnose fruit rot (AFR) and Botrytis fruit rot (BFR), caused by Colletotrichum acutatum and Botrytis cinerea, respectively, are among the most important fruit rot diseases. Strawberry growers tend to be risk-averse and traditionally rely on weekly preventative applications of fungicides for control of these diseases. In Florida, the Strawberry Advisory System (StAS) has been developed to forecast the need for fungicide applications based on environmental conditions and previously developed disease models. The first prototype of this decision support system was released in 2009. Subsequently, close to 40 commercial field trials were set up over four years to determine the effectiveness of StAS for AFR and BFR management compared to the traditional calendar-based spray program. A meta-analysis was conducted to quantify the treatment effects on BFR incidence, AFR incidence, yield, and the number of marketable fruit. No differences were observed between StAS and calendar-based treatments for any of the treatment effects. However, the number of fungicide applications was reduced by a median of $50 \%$ when following the advisory system compared with the calendar-based approach. The commercial field trials, as well as recent challenges with increased fungicide resistance, have aided in the adoption of the system by Florida strawberry growers. However, nuances in the way the system is used in the reality of a high-value, high-risk crop have arisen and will be discussed. 